\title{
Cyclic quantum evolution and Aharonov-Anandan geometric phases in SU(2) spin-coherent states
}

\author{
Eric Layton, Youhong Huang, ${ }^{*}$ and Shih-I Chu \\ Department of Chemistry, University of Kansas, Lawrence, Kansas 66045
}

(Received 5 June 1989)

\begin{abstract}
We show that cyclic quantum evolution can be realized and the Aharonov-Anandan (AA) geometric phase can be determined for any spin- $j$ system driven by periodic fields. Two methods are extended for the study of this problem: the generalized spin-coherent-state technique and the Floquet quasienergy approach. Using the former approach, we have developed a generalized Bloch-sphere model and presented a SU(2) Lie-group formulation of the AA geometric phase in the spin-coherent state. We show that the AA phase is equal to $j$ times the solid angle enclosed by the trajectory traced out by the tip of a generalized Bloch vector. General analytic formulas are obtained for the Bloch vector trajectory and the AA geometric phase in terms of external physical parameters. In addition to these findings, we have also approached the same problem from an alternative but complementary point of view without recourse to the concept of coherent-state terminology. Here we first determine the Floquet quasienergy eigenvalues and eigenvectors for the spin- $j$ system driven by periodic fields. This in turn allows the construction of the time-evolution propagator, the total wave function, and the AA geometric phase in a more general fashion.
\end{abstract}

\section{INTRODUCTION}

Considerable interest has been generated by the recent discovery by Berry ${ }^{1}$ regarding the geometrical phase factor associated with the adiabatic transport of a quantum system around a closed circuit in some parameter space. ${ }^{2}$ In addition to the "normal" dynamical phase

$$
\alpha_{D}=-\frac{i}{\hbar} \int\langle\psi(t)|\hat{H}(t)| \psi(t)\rangle d t
$$

a geometric phase factor $\beta_{G}(C)$, now known as the Berry's phase, evolves during the cyclic evolution, which depends only upon the geometry of the circuit $C$. Several experiments have already been reported that demonstrate the effects of this phase. These include observations on nuclear magnetic resonance ${ }^{3}$ (NMR), photons, ${ }^{4}$ neutrons, ${ }^{5}$ nuclear spins, ${ }^{6}$ molecular energy levels, ${ }^{7}$ and electrons, ${ }^{8}$ etc. More recently, Aharonov and Anandan ${ }^{9}$ (AA) have introduced a new cyclic quantum phase that is a gauge-invariant generalization of the Berry phase without recourse to adiabaticity. The AA phase is a more general concept ${ }^{10}$ and is associated with the evolution of any cyclic state, i.e., a quantum state $|\psi(t)\rangle$ which returns to itself, apart from a phase factor, after some time $T: \quad|\psi(T)\rangle=\exp (i \Phi)|\psi(0)\rangle$, where $\Phi$ (total phase $)=\alpha_{D}$ (dynamical phase $)+\beta_{G}$ (geometric phase). The AA geometric phase $\beta_{G}$ is related to an holonomy ${ }^{2}$ associated with parallel transport around the circuit in projective Hilbert space. The importance of the AA formulation is that it applies whether or not the Hamiltonian $\hat{H}(t)$ is cyclic or adiabatic. The AA geometric phase only depends upon the cyclic evolution of the system. This establishes a simple connection of the geometric phase to the Aharonov-Bohm effect ${ }^{11}$ which does not invoke the adiabiticity of the circuit. The AA phase has also been recently detected experimentally by means of NMR interferometry. ${ }^{12}$
In this paper we consider the Aharonov-Anandan geometric phases for the cyclic quantum evolution of any spin $j$ driven by periodic fields. We note that while Berry's phase for spin motion has been extensively studied, ${ }^{1,13}$ to our knowledge, the current work is the first general and extensive treatment of AA phases in the cyclic quantum evolution of any spin $j$ system. In general, for any $N$-level system [subject to $\mathrm{SU}(N)$ dynamical symmetry], the cyclic evolution of the quantum state $\psi(t)$ is difficult to realize if $N \geq 3$ since there are $N^{2}-1$ degrees of freedom in the Hilbert space. However, we shall show that for the spin- $j$ system, it is feasible to satisfy the cyclic condition and that the AA phase can be measured experimentally. Two methods will be presented for the treatment of this problem: the generalized coherent-state technique $^{14}$ and the Floquet quasienergy approach. ${ }^{15}$

In Sec. II, we review briefly the SU(2) Lie-group properties of the spin-coherent states. We have chosen a coordinate system somewhat different from that of Perelomov $^{14}$ so that our results can be easily extended to both NMR and quantum optics. In Sec. III, we present a Lie-group formulation of the AA geometric phase in the spin-coherent state. We show that the AA phase is equal to $j$ times the solid angle enclosed by the trajectory traced out by the tip of a generalized Bloch vector. General analytic formulas are presented for the geometric phases in terms of external physical parameters. In Sec. IV, we present an alternative approach, the Floquet quasienergy formalism, for the study of AA geometric phases in spin systems driven by periodic fields. Here we first determine the quasienergy eigenvalues and eigenvectors of the spin- $j$ systems. This allows the construction of the time-evolution operator, the total wave function, and then the AA geometric phases in a general fashion without recourse to the concept of coherent-state language. Finally, we present a conclusion and outlook in Sec. V. 


\section{SU(2) SPIN-COHERENT STATES}

The quantum evolution of a particle with spin $j$ and magnetic moment $\mu$ in a variable magnetic field $\mathbf{B}(t)=\left(B_{x}(t), B_{y}(t), B_{z}(t)\right)$ is governed by the timedependent Schrödinger equation $(\hbar=1)$

$$
i \frac{d}{d t}|\psi(t)\rangle=\hat{H}(t)|\psi(t)\rangle \text {. }
$$

Here $\hat{H}(t)$ is the Hamiltonian

$$
\hat{H}(t)=-\boldsymbol{\mu} \cdot \widehat{\mathbf{B}}(t)=-(\mu / j) \mathbf{J} \cdot \mathbf{B},
$$

and $\mathbf{J}=\left(\hat{J}_{x}, \hat{J}_{y}, \hat{J}_{z}\right)$ is the spin angular momentum operator with $\hat{J}_{i}$ corresponding to the operator of infinitesimal rotation around the $i$ th axis $(i=x, y, z)$. It is known that the spin motion can be described by the SU(2) group, ${ }^{14}$ as the Hamiltonian, Eq. (2), contains only the generators of the $\mathrm{SU}(2)$ group

$$
\hat{H}(t)=i\left[a(t) \hat{J}_{+}-a^{*}(t) \widehat{J}_{-}-i b(t) \widehat{J}_{0}\right],
$$

where

$$
\begin{aligned}
& a(t)=i(\mu / 2 j)\left[B_{x}(t)-i B_{y}(t)\right], \\
& b(t)=-(\mu / j) B_{z}(t),
\end{aligned}
$$

and the infinitesimal operators $\hat{J}_{ \pm}=\widehat{J}_{x} \pm i \hat{J}_{y}, \widehat{J}_{0}=\hat{J}_{z}$ obey the commutation relations

$$
\left[\hat{J}_{0}, \hat{J}_{ \pm}\right]= \pm \widehat{J}_{ \pm},\left[\hat{J}_{-}, \hat{J}_{+}\right]=-2 \widehat{J}_{0} \text {. }
$$

Any unitary irreducible representation $T(g)$ of the group $\mathrm{SU}(2)$ is given by a non-negative integer or half-integer $j$ : $T(g)=T^{j}(g)$ with dimension $(2 j+1)$. The representation space vectors $|j, m\rangle$ are eigenvectors of the operators $\hat{J}_{0}$ and $\mathbf{J}^{2}\left(=\widehat{J}_{x}^{2}+\widehat{J}_{y}^{2}+\widehat{J}_{z}^{2}\right)$

$\widehat{J}_{0}|j, m\rangle=m|j, m\rangle$,

$\mathbf{J}^{2}|j, m\rangle=j(j+1)|j, m\rangle$

$$
(m=-j,-j+1, \ldots, j) .
$$

The time evolution in Eq. (1) can be solved most expediently by the use of spin-coherent-state (CS) terminology. ${ }^{14}$ If a vector $|j, m\rangle$ is taken as the fundamental vector $\left|\psi_{0}\right\rangle$, the coherent state is determined by a unit vector $\widehat{\mathbf{n}}=(\sin \theta \cos \phi, \sin \theta \sin \phi, \cos \theta)$ :

$$
|\widehat{\mathbf{n}}\rangle=D(\widehat{\mathbf{n}})\left|\psi_{0}\right\rangle=T^{j}\left(g_{\widehat{\mathbf{n}}}\right)\left|\psi_{0}\right\rangle \text {. }
$$

That is, the coherent state $|\hat{\mathbf{n}}\rangle$ corresponds to a point of the two-dimensional sphere $S^{2}=\mathrm{SO}(3) / \mathrm{SO}(2)=\mathrm{SU}(2) /$ $\mathrm{U}(1)$ with unit radius. In principle, any vector $|j, m\rangle$ with arbitrary $m$ can be considered as the fundamental vector $\left|\psi_{0}\right\rangle$. In practice, only the vectors $|j, \pm j\rangle$ give rise to the minimum dispersion for the Casimir operator $\widehat{\mathbf{J}}^{2}$. Thus the spin-coherent states determined by $\left|\psi_{0}\right\rangle=|j, \pm j\rangle$ are the closest to the classical states. In the following we shall choose the ground state $|j,-j\rangle$ as the fundamental vector $\left|\psi_{0}\right\rangle$ corresponding to the south pole point $\widehat{\mathbf{n}}_{0}=(0,0,-1)$ in the two-dimensional sphere.

Figure 1 shows the relation between $\hat{\mathbf{n}}$ and $\hat{\mathbf{n}}_{0}$. The coherent state $|\hat{\mathbf{n}}\rangle$ is related to $\left|\hat{\mathbf{n}}_{0}\right\rangle$ by three successive rotations

$$
\begin{aligned}
|\hat{\mathbf{n}}\rangle= & \exp \left[i(\pi / 2-\phi) \hat{J}_{z}\right] \exp \left[-i(\pi-\theta) \hat{J}_{x}\right] \\
& \times \exp \left[-i(\pi / 2-\phi) \hat{J}_{z}\right]\left|\hat{\mathbf{n}}_{0}\right\rangle \\
= & \exp \left[\kappa \hat{J}_{+}-\kappa^{*} \hat{J}_{-}\right]\left|\hat{\mathbf{n}}_{0}\right\rangle
\end{aligned}
$$

where $\kappa=[(\pi-\theta) / 2] e^{-i \phi}$. Using the Gaussian decomposition method, ${ }^{16} \mathrm{Eq}$. (8b) can be rewritten as

$$
|\xi\rangle \equiv|\hat{\mathbf{n}}\rangle=\exp \left(\xi \widehat{J}_{+}\right) \exp \left(\eta \widehat{J}_{0}\right) \exp \left(\xi^{\prime} \widehat{J}_{-}\right)|j,-j\rangle,
$$

where

$$
\begin{aligned}
& \xi=\kappa \sin |\kappa| /(|\kappa| \cos |\kappa|)=\cot (\theta / 2) e^{-i \phi}, \\
& \eta=\ln \left(1+|\xi|^{2}\right),
\end{aligned}
$$

and

$$
\xi^{\prime}=-\xi^{*} .
$$

Equations (9) and (10) show that the coherent state $|\xi\rangle$ or $|\hat{\mathbf{n}}\rangle$ may be parametrized by a complex number $\xi$. Substituting Eq. (10b) into Eq. (9), we get

$$
\begin{aligned}
|\xi\rangle & =\left(1+|\xi|^{2}\right)^{-j} \exp \left(\xi \hat{J}_{+}\right)|j,-j\rangle \\
& =\sum_{m=-J}^{j} C_{m}(\xi)|j, m\rangle,
\end{aligned}
$$

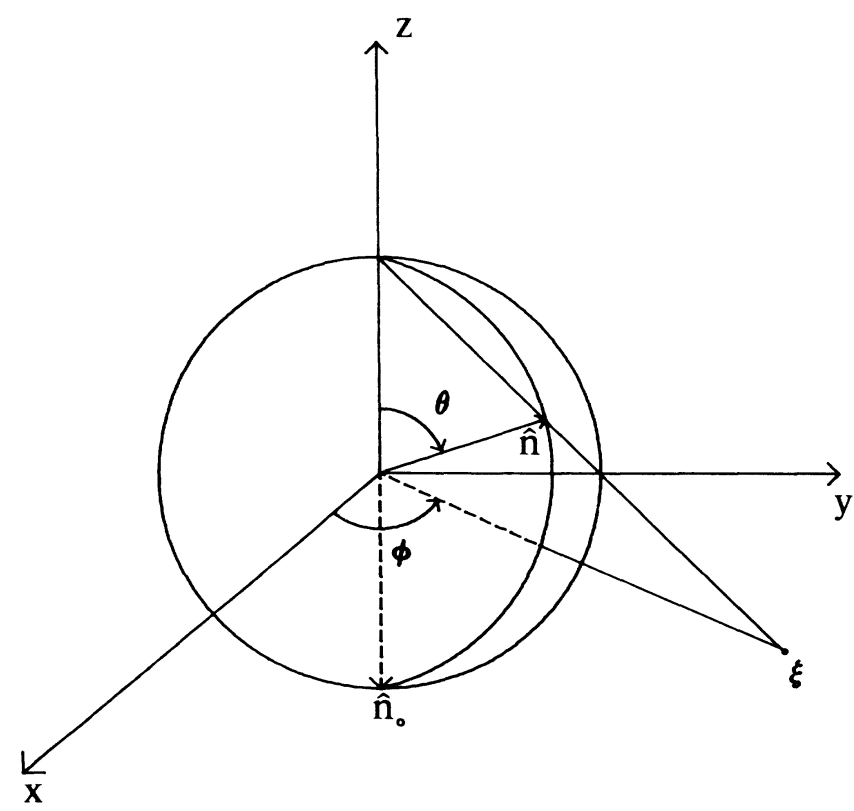

FIG. 1. Generalized Bloch sphere model for any spin $j$. Each point $\widehat{\mathbf{n}}=(\sin \theta \cos \phi, \sin \theta \sin \phi, \cos \theta)$ on the (unit radius) Bloch sphere represents a spin-coherent state. The unit vector $\widehat{\mathbf{n}}_{0}$ (south pole) represents the fundamental vector $\left|\psi_{0}\right\rangle=|j,-j\rangle$. Also shown here is the mapping of a three-dimensional unit vector $\hat{\mathbf{n}}$ to a point $\xi$ (complex parameter) in the $x y$ plane. 
with

$C_{m}(\xi)=\left(\frac{(2 j) !}{(j+m) !(j-m) !}\right)^{1 / 2}\left(1+|\xi|^{2}\right)^{-\jmath} \xi^{j+m}$

Figure 1 shows the mapping of a three-dimensional unit vector $\hat{\mathbf{n}}$ to the parameter $\xi$ in the $x y$ plane, defined by Eq. (10a).

\section{AA GEOMETRIC PHASES \\ IN SPIN-COHERENT STATES}

\section{A. Equation of motion for spin-coherent states under cyclic evolution}

Under a cyclic quantum evolution,

$$
|\psi(t+T)\rangle=e^{i \Phi}|\psi(t)\rangle
$$

and

$$
\hat{\rho}(t+T)=\hat{\rho}(t),
$$

where $\Phi$ is the total phase associated with the cyclic evolution of $|\psi(t)\rangle$ and $\hat{\rho}$ is the density-matrix operator

$$
\hat{\rho}(t)=|\psi(t)\rangle\langle\psi(t)| .
$$

To evaluate $\Phi$, we follow the procedure of Aharonov and Anandan ${ }^{9}$ by setting

$$
|\psi(t)\rangle=e^{i f(t)}|\xi(t)\rangle
$$

where $f(t)$ is an arbitrary function satisfying $f(t+T)-f(t)=\Phi$, and

$$
|\xi(t+T)\rangle=|\xi(t)\rangle .
$$

Substituting Eq. (15) into the Schrödinger equation, Eq. (1), we obtain the equations of motion for $|\xi(t)\rangle$ and $f(t)$

$$
i \frac{d}{d t}|\xi(t)\rangle=[\hat{H}(t)+\dot{f}]|\xi(t)\rangle
$$

and

$$
\frac{d f}{d t}=\left\langle\xi\left|i \frac{\partial}{\partial t}\right| \xi\right\rangle-\langle\xi|\hat{H}| \xi\rangle .
$$

From Eq. (18), we find

$$
\Phi=\alpha_{D}+\beta_{G}
$$

where the dynamical phase

$$
\alpha_{D}=-\int_{0}^{T}\langle\psi|\hat{H}| \psi\rangle d t
$$

and the AA geometric phase

$$
\beta_{G}=\int_{0}^{T}\left\langle\xi\left|i \frac{\partial}{\partial t}\right| \xi\right\rangle d t .
$$

Before proceeding with the evaluation of the phases, we note the following relevant formulas for spin-coherent states: ${ }^{14}$

$$
\begin{aligned}
& \hat{J}_{+}|\xi\rangle=\xi^{-1}\left(j+\widehat{J}_{0}\right)|\xi\rangle, \\
& \hat{J}_{-}|\xi\rangle=\xi\left(j-\hat{J}_{0}\right)|\xi\rangle
\end{aligned}
$$

and

$\frac{d}{d t}|\xi(t)\rangle=\left[\frac{-j}{1+|\xi|^{2}} \frac{d}{d t}\left(1+|\xi|^{2}\right)+\dot{\xi} \hat{J}_{+}\right)|\xi(t)\rangle$.

Equation (24) can be obtained directly by differentiating Eq. (11) with respect to time. Substituting Eq. (3) into Eq. (17) and using Eqs. (22)-(24), we obtain the following equations of motion for the parameters $\xi(t)$ and $f(t)$ :

$$
\dot{\xi}(t)=a(t)+a^{*}(t) \xi^{2}(t)-i b(t) \xi(t)
$$

and

$$
-i \dot{f}(t)=j\left[-a(t) \xi^{*}(t)+a^{*}(t) \xi(t)-i b(t)\right] .
$$

Hence the quantum time-evolution problem now reduces to the solution of simpler classical time evolution of $\xi(t)$ and $f(t)$. Note that Eq. (25) has the form of a Ricatti equation.

\section{B. AA geometric phase and solid angle theorem}

We can now proceed to evaluate the AA geometric phase $\beta_{G}$. From Eqs. (21) and (24), we have

$$
\begin{aligned}
\beta_{G} & =i \int_{0}^{T}\langle\xi \mid \dot{\xi}\rangle d t \\
& =i \int_{0}^{T}\left[\frac{-j}{1+|\xi|^{2}} \frac{d}{d t}\left(1+|\xi|^{2}\right)+\dot{\xi}\left\langle\xi\left|\hat{J}_{+}\right| \xi\right\rangle\right) d t
\end{aligned}
$$

Using the group property of the SU(2) coherent states,

$$
\left\langle\xi\left|\hat{J}_{+}\right| \xi\right\rangle=2 j \frac{\xi^{*}}{1+|\xi|^{2}},
$$

and noticing $|\xi(0)|=|\xi(T)|$, we find

$$
\beta_{G}=-2 j \int_{0}^{T} \frac{\left(\xi_{1} d \xi_{2}-\xi_{2} d \xi_{1}\right)}{1+|\xi|^{2}},
$$

where we have set $\xi=\xi_{1}+i \xi_{2}$. The integral can be evaluated explicitly by using Eq. (10a), yielding [to $\bmod (2 \pi)$ ]

$$
\beta_{G}=-j \int_{C}(1-\cos \theta) d \phi,
$$

where $C$ is the closed loop traced by the vector $\widehat{\mathbf{n}}(t)$. Equation (29) is a main result of this paper which states that the AA geometric phase $\beta_{G}$ for spin- $j$ systems with Hamiltonians of the form - $\widehat{\mathbf{B}} \cdot \widehat{\mathbf{J}}$ [Eq. (2)] is equal to $j$ times the solid angle $\Omega(C) \equiv \int_{C}(1-\cos \theta) d \phi$ enclosed by the trajectory $\hat{\mathbf{n}}(t)$. For the special case $j=\frac{1}{2}$, Eq. (29) reduces to the known result. ${ }^{17}$

\section{Solution of the Ricatti equation and the AA geometric phase for the case of rotating magnetic field}

We now consider the solution of the Ricatti equation, Eq. (25), for the trajectory of the complex coherent-state parameter $\xi(t)$. Equation (25) can be linearized by substituting

$$
\xi(t)=-\frac{\dot{x}}{a^{*} x}=-\frac{1}{a^{*}} \frac{d}{d t} \ln x,
$$

yielding 


$$
\ddot{x}+\left(i b-\dot{a}^{*} / a^{*}\right) \dot{x}+|a|^{2} x=0 .
$$

Introducing the variable $y=\dot{x}$, we obtain a set of two first-order coupled differential equations

$$
\begin{aligned}
\frac{d}{d t}\left(\begin{array}{l}
x \\
y
\end{array}\right] & =\left(\begin{array}{cc}
0 & 1 \\
-|a|^{2} & -\left(i b-\dot{a}^{*} / a^{*}\right)
\end{array}\right)\left(\begin{array}{l}
x \\
y
\end{array}\right) \\
& =\hat{A}(t)\left(\begin{array}{l}
x \\
y
\end{array}\right) .
\end{aligned}
$$

An important class of problems of particular interest to NMR experiments and quantum optics involves periodically driven fields where $\hat{A}(t)$ is periodic in time. Floquet theory ${ }^{15}$ can be invoked to facilitate the study of Eq. (30). Here we shall consider only the special but important case of rotating magnetic field $\mathbf{B}=\left(B \cos \omega t, B \sin \omega t, B_{0}\right)$, where $\xi(t)$ can be solved in closed form. In this case, $a=i a_{0} e^{-i \omega t}, a_{0}=\mu B / 2 j, b=-\mu B_{0} / j$, and $\hat{A}$ is time independent:

$$
\hat{A}=\left[\begin{array}{cc}
0 & 1 \\
-a_{0}^{2} & -i\left(\omega_{0}-\omega\right)
\end{array}\right),
$$

with $\omega_{0}=b$. We find

$$
\xi(t)=\left(\frac{i a_{0} \sin (\Omega t / 2)-\xi_{0}[(-\Omega / 2) \cos (\Omega t / 2)+i(\Delta / 2) \sin (\Omega t / 2)]}{(\Omega / 2) \cos (\Omega t / 2)+i(\Delta / 2) \sin (\Omega t / 2)+i a_{0} \xi_{0} \sin (\Omega t / 2)}\right) e^{-i \omega t},
$$

with the initial condition $\xi(0)=\xi_{0}$. Here $\Omega$ is the Rabi frequency and $\Delta$ the detuning

$$
\Omega=\left(\Delta^{2}+4 a_{0}^{2}\right)^{1 / 2}, \quad \Delta=\omega_{0}-\omega .
$$

Equation $(31)$ is the trajectory of $\xi(t)$ in the laboratory frame. One sees that after the period $T=2 \pi / \Omega$, the parameter $\xi(t)$ returns to the initial point $\xi_{0}$ apart from the phase $e^{-i \omega T}$. This phase factor can be eliminated by transforming the equation of motion into the so-called rotating frame [i.e., the frame rotating with the magnetic field $\mathbf{B}(t)$ ]. The result is

$$
\xi_{R}(t)=\xi(t) e^{i \omega t}
$$

Thus $\xi_{R}(t)$ is a periodic function of time with period $T$, and the coherent state $\left|\xi_{R}(t+T)\right\rangle$ is guaranteed to return to $\left|\xi_{R}(t)\right\rangle$ in the rotating frame.

We can now proceed to evaluate the AA geometric phase. We shall first choose the initial state of the system starting from the ground state $|j,-j\rangle$ or, equivalently, from the south pole $\hat{\mathbf{n}}_{0}=(0,0,-1)$ in the CS sphere. In this case, $\xi_{0}=0$ and

$$
\xi_{R}(t)=\frac{i a_{0} \sin (\Omega t / 2)}{(\Omega / 2) \cos (\Omega t / 2)+i(\Delta / 2) \sin (\Omega t / 2)} .
$$

Substituting Eqs. (34) and (10a) into Eq. (29) and performing the integration using the Riemann-Stieljes integral technique, ${ }^{18}$ we arrive at the AA geometric phase

$$
\beta_{G}=-2 \pi j(1+\Delta / \Omega),
$$

expressed directly in terms of the experimental parameters $\Delta$ and $\Omega$. For the case $j=\frac{1}{2}$, Eq. (35) reduces to the known result we have obtained earlier. ${ }^{17}$

For the initial state starting from an arbitrary point on the (coherent-state) unit sphere $\widehat{\mathbf{n}}_{0}$ $=\left(\sin \theta_{0} \cos \phi_{0}, \sin \theta_{0} \sin \phi_{0}, \cos \theta_{0}\right) \quad$ corresponding to the mapping point $\xi_{0}=\cot \left(\theta_{0} / 2\right) \exp \left(-i \phi_{0}\right)$ in the $x y$ plane, the above procedure is tedious to perform. We shall instead pursue a different route using the generalized Bloch vector model described in Sec. II D. The procedure is more elegant and direct.

\section{Generalized Bloch vector model and the AA geometric phase}

Consider again the Ricatti equation, Eq. (25). Using the relation between the parameter $\xi$ and $(\theta, \phi)$ defined in Eq. (10a), we can derive the following equations of motion for $\theta(t)$ and $\phi(t)$ :

$$
\begin{aligned}
& \dot{\theta}=2(\operatorname{Im} a) \sin \phi-2(\operatorname{Re} a) \cos \phi, \\
& \dot{\phi}=b+2(\operatorname{Im} a) \cos \phi \cot \theta+2(\operatorname{Re} a) \sin \phi \cot \theta .
\end{aligned}
$$

From these, we find the equation of motion for the unit vector $\hat{\mathbf{n}}=(\sin \theta \cos \phi, \sin \theta \sin \phi, \cos \theta)$ :

$$
\dot{\hat{\mathbf{n}}}(t)=\mathbf{\Omega}(t) \times \hat{\mathbf{n}}(t),
$$

where $\boldsymbol{\Omega}=(-2 \operatorname{Im} a,-2 \operatorname{Re} a, b)$. This equation is identical in form to the celebrated Bloch vector model for the spin $-\frac{1}{2}$ system. $^{19}$ Equation (37), valid for any spin $j$, can thus be considered as the generalized Bloch vector model. The unit vector $\hat{\mathbf{n}}$ can thus be identified as the generalized Bloch vector whose tip traces out a trajectory on the surface of the generalized Bloch sphere. Each point on the sphere represents a spin-coherent state. Similar to the spin $-\frac{1}{2}$ case, the generalized Bloch vector for spin $j$ contains three components

$$
\widehat{\mathbf{n}} \equiv(u, v, w) \text {, }
$$

where

$$
\begin{aligned}
& u(t)=\operatorname{Tr}\left[\hat{\rho}(t) \hat{J}_{x}\right]=\sin \theta \cos \phi, \\
& v(t)=\operatorname{Tr}\left[\hat{\rho}(t) \hat{J}_{y}\right]=\sin \theta \sin \phi, \\
& w(t)=\operatorname{Tr}\left[\hat{\rho}(t) \widehat{J}_{z}\right]=\cos \theta,
\end{aligned}
$$

with

$$
u^{2}(t)+v^{2}(t)+w^{2}(t)=1 .
$$

To achieve a cyclic evolution for the spin-coherent state $|\hat{\mathbf{n}}\rangle \equiv|\xi\rangle$, we need to consider the equation of motion in the rotating frame defined by the transformation 


$$
\hat{R}=\left(\begin{array}{ccc}
\cos \omega t & \sin \omega t & 0 \\
-\sin \omega t & \cos \omega t & 0 \\
0 & 0 & 1
\end{array}\right)
$$

The result is

$$
\dot{\hat{\mathbf{n}}}_{R}=\hat{\mathbf{\Omega}}_{R} \times \widehat{\mathbf{n}}_{R},
$$

where

$$
\widehat{\mathbf{n}}_{R}=\hat{\mathbf{n}} \hat{R}^{T},
$$

and $\omega$ is the frequency of the driving field. In the case of the rotating magnetic field $\mathbf{B}=\left(B \cos \omega t, B \sin \omega t, B_{0}\right), \boldsymbol{\Omega}_{R}$ becomes time independent

$$
\boldsymbol{\Omega}_{R}=\left(-2 a_{0}, 0, \Delta\right),
$$

and Eq. (41) can be solved in closed form for $\widehat{\mathbf{n}}_{R}(t)$. Note that $\hat{\mathbf{n}}_{R}(t+T)=\hat{\mathbf{n}}_{R}(t)$ and $\hat{\mathbf{n}}_{R}$ traces out a closed loop after an interval $T=2 \pi / \Omega$, where $\Omega=\left|\Omega_{R}\right|=\left(\Delta^{2}\right.$ $\left.+4 a_{0}^{2}\right)^{1 / 2}$. Equation (41) provides a classical precession picture for the motion of $\hat{\mathbf{n}}_{R}(t)$. Thus, starting from an initial point on the generalized Bloch sphere

$$
\widehat{\mathbf{n}}_{R}(0)=\left(\sin \theta_{0} \cos \phi_{0}^{R}, \sin \theta_{0} \sin \phi_{0}^{R}, \cos \theta_{0}\right),
$$

the vector $\hat{\mathbf{n}}_{R}(t)$ precesses around the fixed vector $\boldsymbol{\Omega}_{R}$ in time with the angle $\gamma$ between $\widehat{\mathbf{n}}(t)$ and $\boldsymbol{\Omega}_{R}$ being held constant:

$$
\gamma=\cos ^{-1}\left[\boldsymbol{\Omega}_{R} \cdot \hat{\mathbf{n}}_{R}(0) /\left|\boldsymbol{\Omega}_{R}\right|\right] .
$$

The AA geometric phase can now be readily obtained from Eq. (29),

$$
\beta_{G}=-2 \pi j(1-\cos \gamma) .
$$

Substituting Eqs. (42) and (43) into Eq. (44), we have the general formula

$$
\beta_{G}=-2 \pi j\left(1+\frac{2 a_{0} \sin \theta_{0} \cos \phi_{0}^{R}-\Delta \cos \theta_{0}}{\left(\Delta^{2}+4 a_{0}^{2}\right)^{1 / 2}}\right) .
$$

For the special initial condition $\hat{\mathbf{n}}_{R}(0)=(0,0,-1)$, the south pole of the Bloch sphere, we have $\theta_{0}=\pi$, and Eq. (45b) reduces to the previous result, Eq. (35), obtained in Sec. II C.

\section{GEOMETRIC PHASES IN SPIN SYSTEMS DRIVEN BY PERIODIC FIELDS: FLOQUET QUASIENERGY APPROACH}

The quantum evolution of the spin- $j$ system, Eq. (1), can also be studied directly via the determination of the time-evolution propagator $\hat{U}\left(t, t_{0}\right)$ without resorting to the use of spin-CS concept. The time-propagator method is more general and can also be applied to nonspin systems. In the case of periodically driven systems, Floquet theories ${ }^{15}$ can be extended for facilitating the solution of the time-dependent Schrödinger equation. The method and results presented in this section complement those shown in Sec. III.

First consider the motion of a spin- $j$ system under the influence of a static magnetic field $(\| \widehat{\mathbf{z}})$ and a linearly po- larized magnetic field in the $x$ direction, as in typical NMR experiments. ${ }^{19}$ Thus

$$
\mathbf{B}(t)=\left(B_{x}^{0} \cos \omega t, 0, B_{z}^{0}\right) .
$$

The Hamiltonian, Eq. (2), now reads

$$
\hat{H}(t)=\omega_{0} \widehat{J}_{z}+4 \omega_{\perp} \hat{J}_{x} \cos \omega t,
$$

where

$$
\omega_{0}=-\mu B_{z}^{0} / j, \quad \omega_{1}=-\mu B_{x}^{0} / 4 j .
$$

As the Hamiltonian $\hat{H}(t)=\hat{H}(t+2 \pi / \omega)$ is periodic in time, the time-dependent Schrödinger equation, Eq. (1), can be transformed into an equivalent time-independent infinite-dimensional Floquet Hamiltonian eigenvalue analysis. $^{15,20}$ We first introduce the Floquet state basis $|j m ; \mu\rangle=|j m\rangle \otimes|\mu\rangle$, where $|j m\rangle$ are the (unperturbed) spin eigenfunctions $(m=-j,-j+1, \ldots, j)$ and $|\mu\rangle$ are the Fourier vectors $(\mu=0, \pm 1, \pm 2, \ldots)$ such that $\langle t \mid \mu\rangle=\exp (i \mu \omega t)$. In terms of the basis $|j m ; \mu\rangle$, the time-independent Floquet Hamiltonian $\hat{H}_{F}$ has the following matrix form:

$$
\begin{aligned}
\left\langle j m ; \mu\left|\hat{H}_{F}\right| j n ; v\right\rangle & \\
=\left(n \omega_{0}+v \omega\right) \delta_{m n} \delta_{\mu v}+ & 2 \omega_{\perp}\left\langle j m ; \mu\left|\hat{J}_{x}\right| j n ; v\right\rangle \\
& \times\left(\delta_{\mu, v+1}+\delta_{\mu, v-1}\right),
\end{aligned}
$$

where $\mu$ and $v$ are Fourier indices $(-\infty$ to $+\infty)$. The procedure is to solve the time-independent eigenproblem

$$
\hat{H}_{F}\left|\varepsilon_{m v}^{(\jmath)}\right\rangle=\varepsilon_{m v}^{(\jmath)}\left|\varepsilon_{\mu v}^{(\jmath)}\right\rangle .
$$

Here $\varepsilon_{m v}^{(\jmath)}$ and $\left|\varepsilon_{m v}^{(j)}\right\rangle$ are, respectively, the quasienergy eigenvalues and eigenvectors, and they satisfy the following periodicity relations:

$$
\begin{aligned}
& \varepsilon_{m v}^{(j)}=\varepsilon_{m 0}^{(j)}+v \omega, \\
& \left\langle j n, \mu+p \mid \varepsilon_{m, v+p}^{(j)}\right\rangle=\left\langle j n, \mu \mid \varepsilon_{m, v}^{(j)}\right\rangle .
\end{aligned}
$$

The time propagator can be constructed from

$$
\hat{U}\left(t, t_{0}\right)=\sum_{m=-\jmath}^{\jmath} \sum_{-=-\infty}^{\infty}\left|\varepsilon_{m v^{\prime}}^{(j)}\right\rangle \exp \left[-i \varepsilon_{m v^{\prime}}^{(j)}\left(t-t_{0}\right)\right]\left\langle\varepsilon_{m v^{\prime}}^{(j)}\right| \text {. }
$$

In general, there is no closed-form solution for quasienergy eigenvalues and eigenvectors. However, in the rotating-wave approximation ( $\mathrm{RWA}$ ), an exact analytic solution can be obtained for the spin- $j$ problem. This approximation is equivalent to replacing the linearly polarized magnetic field by a rotating field. The RWA is generally valid in NMR conditions where $\left|\Delta / \omega_{0}\right|<<1$ and $\left|\omega_{1} / \omega_{0}\right|<<1$. (Analytical results can still be obtained beyond the RWA limit using high-order nearly degenerate perturbation techniques. ${ }^{21}$ See Ref 17 for an example of the treatment of the high-order multiphoton geometric phase. The more accurate treatment of Floquet eigenvectors will not, however, affect the geometric phase formulation in general.) In the RWA, we drop all energy-nonconserving terms, and the infinite-dimensional Floquet Hamiltonian reduces to block-diagonal RWA Hamiltonians 


$$
\begin{aligned}
\langle j m ; \mu| & \hat{H}_{\mathrm{RWA}}|j n ; v\rangle \\
= & \left(n \omega_{0}+v \omega\right) \delta_{m n} \delta_{\mu v}+\omega_{\perp}\left[\hat{J}_{+}\right]_{m n} \delta_{\mu, v-1} \\
& +\omega_{1}\left[\widehat{J}_{-}\right]_{m n} \delta_{\mu, v+1}
\end{aligned}
$$

with each block being $(2 j+1)$ by $(2 j+1)$ dimensional, where

$$
\left[\widehat{J}_{ \pm}\right]_{m n}=[j(j+1)-n(n \pm 1)]^{1 / 2} \delta_{m, n \pm 1} .
$$

Only one block needs to be considered as all blocks give the identical information. We shall choose the central block defined by the set of Floquet state basis

$$
(|j,-j ; 0\rangle,|j,-j+1 ;-1\rangle, \ldots|j, j ;-2 j\rangle) \text {. }
$$

These are the set of nearly degenerate states coupled by the magnetic dipole interaction. In principle, the $(2 j+1)$-dimensional RWA matrix so defined can be numerically diagonalized. But this procedure does not lead directly to analytic solutions. To achieve this goal, we perform a rotation diagonalization analysis of the (timeindependent) RWA Hamiltonian

$$
\widehat{H}_{\mathrm{RWA}}=-j \omega \hat{I}+\Delta \widehat{J}_{0}+2 \omega_{1} \widehat{J}_{x},
$$

where $\Delta=\omega_{0}-\omega$, and $\hat{I}$ is the identity operator. Notice $\hat{H}_{\text {RWA }}$ possesses the common eigenfunctions as the operator $\hat{h}=\Delta \widehat{J}_{0}+2 \omega_{1} \widehat{J}_{x}$,

$$
\begin{aligned}
& \hat{H}_{\mathrm{RWA}}\left|\varepsilon_{m}\right\rangle=\varepsilon_{m}\left|\varepsilon_{m}\right\rangle, \\
& \hat{h}\left|\varepsilon_{m}\right\rangle=\lambda_{m}\left|\varepsilon_{m}\right\rangle,
\end{aligned}
$$

with $\varepsilon_{m}=\lambda_{m}-j \omega$.

To diagonalize $\hat{h}$, a rotation in the $x z$ plane is necessary. Thus

$$
\begin{aligned}
& \hat{R}(0, \beta, 0)^{-1} \hat{h} \widehat{R}(0, \beta, 0) \\
& \quad=\left(\Delta \cos \beta+2 \omega_{1} \sin \beta\right) \widehat{J}_{0}+\left(-\Delta \sin \beta+2 \omega_{1} \cos \beta\right) \widehat{J}_{x} .
\end{aligned}
$$

To put the right-hand side of Eq. (57) into diagonalized form (in the $|j m\rangle$ basis), the coefficient of the $\widehat{J}_{x}$ operator must vanish

$$
-\Delta \sin \beta+2 \omega_{\perp} \cos \beta=0 .
$$

This leads to

$$
\tan \beta=2 \omega_{1} / \Delta
$$

and

$$
\cos \beta=\Delta /\left(\Delta^{2}+4 \omega_{1}^{2}\right)^{1 / 2}=\Delta / \Omega .
$$

The eigenvalues of $\hat{h}$ in the $|j m\rangle$ basis can now be obtained readily from Eq. (57),

$$
\lambda_{m}=m\left(\Delta \cos \beta+2 \omega_{1} \sin \beta\right)=m \Omega,
$$

from which the quasienergy eigenvalues of $\hat{H}_{\mathrm{RWA}}$ are determined $^{22}$

$$
\varepsilon_{m}=-j \omega+m \Omega \quad(m=-j,-j+1, \ldots, j) .
$$

The quasienergy eigenfunctions, in the rotating frame of coordinates, are

$$
\left|\varepsilon_{m}\right\rangle=\hat{R}(0, \beta, 0)|j m\rangle
$$

or

$$
\left|\varepsilon_{m}\right\rangle=\sum_{m^{\prime}=-j}^{j} d_{m^{\prime} m}^{(j)}(\beta)\left|j m^{\prime}\right\rangle,
$$

where $d_{m^{\prime} m}^{(j)}(\beta)$ are the rotation matrices. ${ }^{23}$

The total wave function for spin- $j$ systems, in rotating frames of coordinates and in RWA, can now be written in closed-form solution

$$
\left|\psi_{R}(t)\right\rangle=\sum_{m=-j}^{j}\left|\varepsilon_{m}\right\rangle\left\langle\varepsilon_{m} \mid \psi(0)\right\rangle e^{-i \varepsilon_{m} t}
$$

or using Eq. (59),

$$
\left|\psi_{R}(t)\right\rangle=e^{i j \omega t} \sum_{m=-j}^{j} e^{-i m \Omega t}\left|\varepsilon_{m}\right\rangle\left\langle\varepsilon_{m} \mid \psi(0)\right\rangle .
$$

Equation (61b) shows that after a period $T=2 \pi / \Omega$, the system returns to $\left|\psi_{R}(0)\right\rangle$ with an extra total overall phase $\Phi$ :

$$
\left|\psi_{R}(T)\right\rangle=e^{i \Phi}\left|\psi_{R}(0)\right\rangle,
$$

and

$$
\Phi=j \omega T-2 \pi j .
$$

The dynamical phase $\alpha_{D}$ is determined by [to $\bmod (2 \pi)$ ]

$$
\begin{aligned}
\alpha_{D} & =-\int_{0}^{T}\left\langle\psi_{R}(t)\left|\hat{H}_{\mathrm{RWA}}\right| \psi_{R}(t)\right\rangle d t \\
& =j \omega T-2 \pi \sum_{m=-j}^{J} m\left|\left\langle\psi(0) \mid \varepsilon_{m}\right\rangle\right|^{2}, \bmod (2 \pi) .
\end{aligned}
$$

Finally, from Eqs. (63) and (64), we find the AA geometric phase $\beta_{G}\left(=\Phi-\alpha_{D}\right)$

$$
\beta_{G}=-2 \pi\left(j-\sum_{m=-j}^{j} m\left|\left\langle\psi(0) \mid \varepsilon_{m}\right\rangle\right|^{2}\right) .
$$

Equation (65) is one of the main results in this paper. The expression is general in that there is no restriction on the initial wave function $|\psi(0)\rangle$. In the event that $|\psi(0)\rangle=|j,-j\rangle$, Eq. $(65)$ reduces to

$$
\begin{aligned}
\beta_{G} & =-2 \pi j(1+\cos \beta) \\
& =-2 \pi j(1+\Delta / \Omega),
\end{aligned}
$$

which is identical to the result, Eq. (35), obtained from the spin-coherent-state approach. Note that in deriving Eq. (66a), use has been made of the relation ${ }^{23}$

$$
\sum_{m=-j}^{j}\left[d_{-j, m}^{(j)}(\beta)\right]^{2}=-j \cos \beta .
$$

As another example, if $\psi(0)=|j, j\rangle$, the result is

$$
\beta_{G}=-2 \pi j(1-\cos \beta) .
$$

This completes the study of the AA geometric phase. 


\section{CONCLUSION}

We have used two different approaches, the spincoherent state and the Floquet quasienergy approach, for studying the feasibility of cyclic quantum evolution of any spin- $j$ system subject to periodic perturbation. We have presented a detailed geometric picture of the spin motion in terms of the generalized Bloch vector model. Further, we have obtained general analytic formulas for the AA geometric phase (directly in terms of external physical parameters) that can be tested by future experiments. We note that while our formalisms are presented only for the spin- $j$ system in terms of NMR terminology, similar ideas and methods can be extended to the study of cyclic evolution in quantum optics ${ }^{24}$.

\section{ACKNOWLEDGMENTS}

This work was supported in part by the U.S. Department of Energy (Division of Chemical Sciences).
*Also at the Department of Physics, University of Kansas, Lawrence, KS 66045.

${ }^{1}$ M. V. Berry, Proc. R. Soc. London, Ser. A 392, 45 (1984).

${ }^{2}$ B. Simon, Phys. Rev. Lett. 51, 2176 (1983).

${ }^{3}$ D. Suter, G. Chingas, R. A. Harris, and A. Pines, Mol. Phys. 61, 1327 (1987).

${ }^{4}$ A. Tomita and R. Y. Chiao, Phys. Rev. Lett. 57, 937 (1986); R. Simon, H. J. Kimble, and E. C. G. Sudarshan, ibid. 61, 19 (1988).

${ }^{5}$ T. Bitter and D. Dubbers, Phys. Rev. Lett. 59, 251 (1987).

${ }^{6}$ R. Tycko, Phys. Rev. Lett. 58, 2281 (1987).

${ }^{7}$ R. S. Ham, Phys. Rev. Lett. 58, 725 (1987); G. Delacretaz, E. R. Grant, R. L. Whetten, L. Woste, and J. W. Zwanziger, ibid. 56, 2598 (1986).

${ }^{8}$ D. M. Bird and A. R. Preston, Phys. Rev. Lett. 61, 2863 (1988).

${ }^{9}$ Y. Aharonov and J. Anandan, Phys. Rev. Lett. 58, 1593 (1987).

${ }^{10}$ See also C. Bouchiat, J. Phys. (Paris) 48, 1627 (1987); C. Bouchiat and G. W. Gibbons, ibid. 49, 187 (1988).

${ }^{11}$ Y. Aharonov and D. Bohm, Phys. Rev. 115, 485 (1959); C. A. Mead and D. G. Truhlar, J. Chem. Phys. 70, 2284 (1979).

${ }^{12}$ D. Suter, K. T. Mueller, and A. Pines, Phys. Rev. Lett. 60, 1218 (1988).

${ }^{13}$ See, for example, T. F. Jordan, J. Math. Phys. 29, 2042 (1988); H. Kuratsuji and S. Iide, Phys. Rev. Lett. 56, 1003 (1986).

${ }^{14}$ A. M. Perelomov, Sov. Phys. Usp. 20, 703 (1977), and references therein.
${ }^{15}$ For recent reviews on Floquet and generalized Floquet methods, see S. I. Chu, Adv. At. Mol. Phys. 21, 97 (1985); Adv. Chem. Phys. 73, 739 (1989).

${ }^{16}$ D. P. Zhelobenko, Compact Lie Groups and Their Representations, Vol. 40 of Translations of Mathematical Monographs (American Mathematical Society, Providence, RI 1973).

${ }^{17}$ S. I. Chu, Z. C. Wu, and E. Layton, Chem. Phys. Lett. 157, 151 (1989).

${ }^{18}$ See, for example, J. M. H. Olmsted, Real Variables (Appleton Century Crofts, New York, 1959).

${ }^{19}$ See, for example, A. Abragam, The Principles of Nuclear Magnetism (Clarendon, Oxford, 1961).

${ }^{20}$ J. H. Shirley, Phys. Rev. 138, 979 (1965).

${ }^{21}$ See, for example, K. Aravind and J. O. Hirschfelder, J. Phys. Chem. 88, 4788 (1984); T. S. Ho and S. I. Chu, J. Phys. B 17, 2101 (1984).

${ }^{22}$ The eigenvalues and eigenfunctions of $\hat{H}_{\mathrm{RWA}}$ can be obtained also by a similar but somewhat different procedure described by R. J. Cook and B. W. Shore, Phys. Rev. A 20, 539 (1979).

${ }^{23}$ L. C. Biedenharn and J. D. Louck, Angular Momentum in Quantum Physics (Addison-Wesley, Reading, MA 1981).

${ }^{24}$ Reference 17 contains a study of the AA phase for two-level system in the optical region. It also includes a generalized density-matrix formulation of complex geometric phases in dissipative systems. 